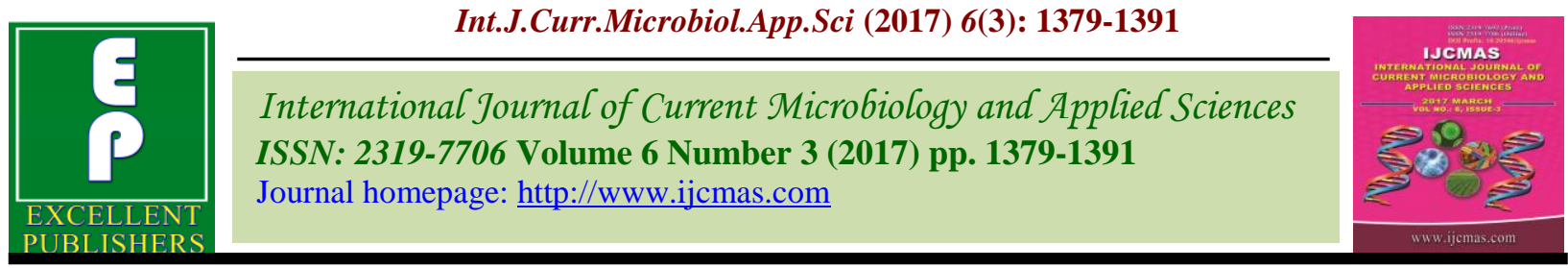

Original Research Article

https://doi.org/10.20546/ijcmas.2017.603.160

\title{
Screening for Alternaria Blight in Clonal Progenies of Potato (Solanum tuberosum L.)
}

\author{
Nirmodh Prabha* and H.C. Nanda \\ Department of Genetics and Plant Breeding, Indira Gandhi KrishiVishwavidyala, SantKabir \\ College of Agriculture and Research Station, Kawardha, India \\ *Corresponding author
}

\section{A B S T R A C T}

Keywords

Alternaria,

Clonal progenies,

Potato, Genotypes.

Article Info

Accepted:

20 February 2017

Available Online:

10 March 2017

\begin{abstract}
18 clonal bulk population, $43 \mathrm{~F}_{1} \mathrm{C}_{2}$ and $90 \mathrm{~F}_{1} \mathrm{C}_{3}$ clonal progenies of potato were screened for resistance against Alternaria solani under natural and artificial conditions in IGKV, Raipur (C.G.). In present study, all the genotypes of clonal bulks, $\mathrm{F}_{1} \mathrm{C}_{2}$ and $\mathrm{F}_{1} \mathrm{C}_{3}$ progenies showed moderate resistance reaction against blight incidence and most of the genotypes of clonal bulks, $\mathrm{F}_{1} \mathrm{C}_{2}$ and $\mathrm{F}_{1} \mathrm{C}_{3}$ clonal progenies showed moderate resistance against blight severity. Among artificial inoculated genotypes of clonal bulks and progenies, the genotype CIP 398201 of clonal bulk, four genotypes (CIP 302024-11-2, CIP 398201-19-1, CIP 398201-19-2 and CIP 398201-20-1) of $\mathrm{F}_{1} \mathrm{C}_{2}$ progenies and five genotypes (CIP 398201-7-2-1, CIP 398201-15-4-1, CIP 398201-5-3-1, CIP 398201-11-6-1 and CIP 398201-2-2-1) of $\mathrm{F}_{1} \mathrm{C}_{3}$ progenies were found moderately resistant against blight incidence. Most of the remaining genotypes showed moderately susceptible reaction.
\end{abstract}

\section{Introduction}

Potato (Solanum tuberosum L.) belongs to Solanaceae family. It is world's leading vegetable crop by virtue of its inherent potential for tonnage production, remunerative income and food values. It encounters a number of serious diseases, with one of the most significant being early blight.

It has been reported that early blight is on the increase in potato growing areas of Europe (Runno-Paurson et al., 2015; Kapsa and Osowski, 2012; Leiminger and Hausladen, 2012). Early blight infections have also been detected with increasing frequency in potato fields in the northern countries of Europe (Edin and Andersson, 2014). Early blight of potato caused by Alternaria solani (Ellis and
Mar.) Jones and Grout, is an important fungal disease, which is widely distributed and damaging.

This pathogen is also known to attack tomatoes, chilies and eggplant. Alternaria blight is potentially a destructive disease of potato (Stuart, 1923) and when present in epiphytotic form can cause defoliation and severe reduction in yield (Whetzel, 1923). It becomes more serious on early-planted crop and becomes a production-limiting factor in some years (Malik and Khan, 1967). So, present studies were designed to screen clonal bulks and progenies of potato against Alternaria blight disease under natural and artificial conditions. 


\section{Materials and Methods}

The experiment were consisted of 18 clonal bulks, $43 \quad \mathrm{~F}_{1} \mathrm{C}_{2}$ and $90 \mathrm{~F}_{1} \mathrm{C}_{3}$ clonal progenies obtained from genetic stock of AICRP on potato, Department of Genetics and Plant Breeding, COA, IGKV, Raipur (C.G.) (Table $1)$.

All potato entries were screened against Alternaria blight under natural (uninoculated) and artificial (inoculated) conditions.

Following observations were recorded on disease incidence under natural condition using following formula.

Disease incidence $(\%)=$

Total number of infected leaves plant ${ }^{-1}$

Total number of leaves plant ${ }^{-1}$

Ten random leaves plant ${ }^{-1}$ were selected to record the disease severity and it was calculated on the basis of per cent leaf area infected.

In addition, three plant of each entries were inoculated artificially by spore suspension of eight days old culture of Alternaria solani, which was first isolated from blight infected plants and identified according to the morphological characteristics of the fungus and then proved the pathogenicity on the potato plants and multiplied on PDA medium and maintained and stored in BOD incubator at $27 \pm 2^{\circ} \mathrm{C}$.

Inoculated plants were covered with polyethylene bags to create favourable conditions for disease development. Observations were taken just after initiation of disease and recorded the disease incidence and severity.

\section{Results and Discussion}

Screening of clonal progenies of potato against Alternaria blight disease

Evaluation of bulks, $F_{1} C_{2}$ and $F_{1} C_{3}$ clonal progenies against Alternaria blight disease under natural conditions

Result of potato bulks are presented in Table 2 indicated that the minimum Alternaria blight incidence was observed in CIP 302431 $(1.70 \%)$ and Kufri Surya (1.70\%) followed by KufriPukhraj (2.10\%), CIP 398203 (2.20\%), CIP 303405 (2.20\%), KufriJawahar (2.20\%), KufriAshoka (3.03\%), CIP 303408 (3.70\%) and CIP 303139 (3.70\%). Alternaria blight incidence was recondded maximum in CIP 302024 (7.70\%) followed by CIP 304146 (7.40\%) and CIP 304124 (6.30\%).

However, in $\mathrm{F}_{1} \mathrm{C}_{2}$ clonal progenies (Table 3) the minimum per cent early blight incidence was observed in KufriPukhraj (1.70\%) followed by CIP 302431-8-1 (2.60\%), KufriJawahar (2.60\%), Kufri Surya (2.70\%), CIP 3980201-20-1 (2.80\%) and CIP 304124$14-1(3.00 \%)$ whereas, the maximum blight incidence was found in CIP 304146-1-1 $(7.80 \%)$ followed by CIP 398203-2-1 (7.50\%), CIP 304146-2-1 (7.30\%), CIP 304146-3-1 (6.70\%) and CIP 302024-10-1 $(6.50 \%)$.

Similarly, the blight incidence in $\mathrm{F}_{1} \mathrm{C}_{3}$ progenies (Table 4) was ranged from 1.00 to 7.70 per cent with the minimum in CIP 303405-5-3-1 (1.00\%) followed by 1.60 per cent incidence was noticed in CIP 304012-62-3 and CIP 303405-6-2-1, 1.80 per cent incidence in CIP 302431-2-1-1 and 1.90 per cent incidence in CIP 303405-3-1-1 and CIP 303405-7-4-1 whereas, maximum per cent incidence was recorded in CIP 304146-2-2-1 (7.70\%) followed by CIP 304124-7-2-1 (7.50\%), CIP 304146-12-4-1 (7.30\%), CIP 
304146-11-2-2 (7.20\%) and CIP 304102-7-1$1(7.10 \%)$.

Based on data per cent disease severity (Table 2). The Alternaria blight disease severity in potato bulks ranged from 3.30 to 15.30 per cent in material studied with a minimum disease severity in CIP 304014 (3.30\%) followed by CIP 302431 (4.60\%), CIP 303405 (5.30\%), CIP 303408 (5.30\%), CIP 398181 (5.30\%), CIP 303139 (5.30\%), KufriJawahar $(5.30 \%)$ and Kufri Surya $(5.30 \%)$. Similarly, disease severity was maximum in CIP $304012(15.30 \%)$ followed by CIP 304102 (12.60\%), CIP 304124 (12.60\%), CIP 302024 (10.60\%), CIP 304146 $(10.60 \%)$ and CIP 398068 (10.0\%). In $\mathrm{F}_{1} \mathrm{C}_{2}$ clonal progenies of potato the blight severity (Table 3) revealed that, the minimum per cent severity was found in entries CIP 303408-10$1(2.80 \%)$ followed by CIP 303405-11-1 (3.30\%) and CIP 398201-20-1 (3.30\%). However, it was recorded maximum in CIP 304146-2-1 (12.60\%) followed by CIP 304014-2-1 (10.0\%) and CIP 303405-13-1, CIP 304014-1-1 and CIP 304124-14-1 (9.30\% in all).

Data on disease severity in $\mathrm{F}_{1} \mathrm{C}_{3}$ clonal progenies ranged from 2.60 to 17.30 per cent (Table 4) in potato. The minimum blight severity percent was noticed in entry CIP 302024-3-1-1 (2.60\%) followed by 3.30 per cent severity recorded in entries viz., CIP 303405-3-1-1, CIP 303405-5-3-1 and CIP 398201-7-2-1 whereas, the entries CIP 398203-4-1-1, CIP 304012-6-2-3, CIP 304014-2-6-1 and CIP 303139-10-3-1 recorded with 4.80 per cent disease severity. Similarly, the per cent of blight severity was recorded the maximum observed in CIP 304146-11-3-1 (17.30\%) followed by CIP 304102-9-3-1 (16.60\%) CIP 304012-1-4-1 (14.60\%) and CIP 303408-2-1-1 (14.0\%).

In present investigation, all the progenies of bulks, $\mathrm{F}_{1} \mathrm{C}_{2}$ and $\mathrm{F}_{1} \mathrm{C}_{3}$ showed moderate resistant reaction against blight incidence (Kang et al., 2007; Kumar et al., 2009). Disease severity in bulks of potato was showed moderate resistant reaction in 13 genotypes and moderate susceptible reaction in five genotypes against blight (Christ and Haynes, 2001; Pandey et al., 2006). Similarly, $42 \mathrm{~F}_{1} \mathrm{C}_{2}$ clonal progenies showed moderately resistance reaction and one showed moderately susceptible resistance against blight. Out of all $\mathrm{F}_{1} \mathrm{C}_{3}$ clonal progenies, 73 progenies exhibited moderate resistant reaction and 17 progenies showed moderate susceptible reaction against Alternaria blight.

Table.1 The Alternaria blight score in per cent was grouped into 1-5 scale (AICRP, Potato)

\begin{tabular}{|c|l|}
\hline Per cent infection & \multicolumn{1}{|c|}{ Category } \\
\hline Above 50.1 & Highly susceptible \\
\hline $25.1-50.0$ & Susceptible \\
\hline $10.1-25.0$ & Moderately susceptible \\
\hline $0.1-10.0$ & Moderately resistant \\
\hline 0.0 & Resistant \\
\hline
\end{tabular}


Table.2 Summary of disease reaction of potato bulks against Alternaria blight diseases

\begin{tabular}{|c|c|c|c|c|c|c|c|}
\hline S. No. & Entries & $\begin{array}{c}\text { Alternaria } \\
\text { blight } \\
\text { incidence } \\
(\%)\end{array}$ & Summary & $\begin{array}{c}\text { Alternaria } \\
\text { blight } \\
\text { severity }(\%)\end{array}$ & Summary & $\begin{array}{c}\text { Alternaria blight } \\
\text { incidence }(\%) \\
\text { (with inoculation) }\end{array}$ & Summary \\
\hline 1 & CIP 398203 & $\begin{array}{c}2.20 \\
(1.64)\end{array}$ & MR & $\begin{array}{c}6.00 \\
(2.55)\end{array}$ & MR & $\begin{array}{c}22.90 \\
(28.60)\end{array}$ & MS \\
\hline 2 & CIP 302024 & $\begin{array}{c}7.70 \\
(2.86)\end{array}$ & MR & $\begin{array}{l}10.60 \\
(3.33)\end{array}$ & MS & $\begin{array}{c}11.60 \\
(19.86)\end{array}$ & MS \\
\hline 3 & CIP 302431 & $\begin{array}{c}1.70 \\
(1.48)\end{array}$ & MR & $\begin{array}{c}4.60 \\
(2.26)\end{array}$ & MR & $\begin{array}{c}13.20 \\
(21.24)\end{array}$ & MS \\
\hline 4 & CIP 304012 & $\begin{array}{c}4.00 \\
(2.12)\end{array}$ & MR & $\begin{array}{l}15.30 \\
(3.97)\end{array}$ & MS & $\begin{array}{c}10.80 \\
(19.16)\end{array}$ & MS \\
\hline 5 & CIP 303405 & $\begin{array}{c}2.20 \\
(1.64)\end{array}$ & MR & $\begin{array}{c}5.30 \\
(2.41)\end{array}$ & MR & $\begin{array}{c}22.10 \\
(27.99)\end{array}$ & MS \\
\hline 6 & CIP 303408 & $\begin{array}{l}3.70 \\
(2.05)\end{array}$ & MR & $\begin{array}{c}5.30 \\
(2.41)\end{array}$ & MR & $\begin{array}{c}15.50 \\
(23.12)\end{array}$ & MS \\
\hline 7 & CIP 304102 & $\begin{array}{c}4.90 \\
(2.32)\end{array}$ & MR & $\begin{array}{l}12.00 \\
(3.54)\end{array}$ & MS & $\begin{array}{c}18.40 \\
(25.31)\end{array}$ & MS \\
\hline 8 & CIP 304146 & $\begin{array}{c}7.40 \\
(2.81)\end{array}$ & MR & $\begin{array}{l}10.60 \\
(3.33)\end{array}$ & MS & $\begin{array}{c}20.90 \\
(27.19)\end{array}$ & MS \\
\hline 9 & CIP 398201 & $\begin{array}{c}4.40 \\
(2.21)\end{array}$ & MR & $\begin{array}{c}6.60 \\
(2.66)\end{array}$ & MR & $\begin{array}{c}7.60 \\
(15.93)\end{array}$ & MR \\
\hline 10 & CIP 304014 & $\begin{array}{c}4.30 \\
(2.19)\end{array}$ & MR & $\begin{array}{c}3.30 \\
(1.95)\end{array}$ & MR & $\begin{array}{l}26.60 \\
(31.05)\end{array}$ & S \\
\hline 11 & CIP 398181 & $\begin{array}{c}4.90 \\
(2.32)\end{array}$ & MR & $\begin{array}{c}5.30 \\
(2.41)\end{array}$ & MR & $\begin{array}{c}12.90 \\
(21.00)\end{array}$ & MS \\
\hline 12 & CIP 304124 & $\begin{array}{c}6.30 \\
(2.61)\end{array}$ & MR & $\begin{array}{l}12.60 \\
(3.62)\end{array}$ & MS & $\begin{array}{c}20.20 \\
(26.67)\end{array}$ & MS \\
\hline 13 & CIP 303139 & $\begin{array}{c}3.70 \\
(2.05)\end{array}$ & MR & $\begin{array}{c}5.30 \\
(2.41)\end{array}$ & MR & $\begin{array}{c}21.90 \\
(27.92)\end{array}$ & MS \\
\hline 14 & CIP 398068 & $\begin{array}{c}4.80 \\
(2.30)\end{array}$ & MR & $\begin{array}{l}10.00 \\
(3.24)\end{array}$ & MR & $\begin{array}{c}21.70 \\
(27.69)\end{array}$ & S \\
\hline 15 & KufriJawahar & $\begin{array}{c}2.20 \\
(1.64)\end{array}$ & MR & $\begin{array}{c}5.30 \\
(2.41)\end{array}$ & MR & $\begin{array}{c}36.70 \\
(37.23)\end{array}$ & MS \\
\hline 16 & KufriAshoka & $\begin{array}{c}3.03 \\
(1.88)\end{array}$ & MR & $\begin{array}{c}8.00 \\
(2.92)\end{array}$ & MR & $\begin{array}{c}23.70 \\
(29.14)\end{array}$ & MS \\
\hline 17 & KufriPukhraj & $\begin{array}{c}2.10 \\
(1.61)\end{array}$ & MR & $\begin{array}{c}7.30 \\
(2.79)\end{array}$ & MR & $\begin{array}{c}16.60 \\
(23.99)\end{array}$ & MS \\
\hline 18 & Kufri Surya & $\begin{array}{r}1.70 \\
(1.48) \\
\end{array}$ & MR & $\begin{array}{r}5.30 \\
(2.41) \\
\end{array}$ & MR & $\begin{array}{c}24.70 \\
(29.77) \\
\end{array}$ & MS \\
\hline & $\begin{array}{l}\text { SEm } \pm \\
\text { C.D. (at 5\%) }\end{array}$ & $\begin{array}{c}0.186 \\
0.54 \\
\end{array}$ & & $\begin{array}{c}0.253 \\
0.73 \\
\end{array}$ & & $\begin{array}{c}1.134 \\
3.26 \\
\end{array}$ & \\
\hline
\end{tabular}

Average of three replications (Data in parenthesis are transformed value)

$\mathrm{MR}=$ Moderately resistant $\mathrm{MS}=$ Moderately susceptible $\mathrm{HR}=$ Highly resistant

$\mathrm{SR}=$ Slightly resistant $\mathrm{S}=$ Susceptible

$\mathrm{ScR}=$ Scarcely resistant 
Table.3 Summary of disease reaction of $\mathrm{F}_{1} \mathrm{C}_{2}$ clonal progenies against Alternaria blight diseases

\begin{tabular}{|c|c|c|c|c|c|c|c|}
\hline S. No. & Entries & $\begin{array}{c}\text { Alternaria } \\
\text { blight } \\
\text { incidence }(\%)\end{array}$ & Summary & $\begin{array}{c}\text { Alternaria } \\
\text { blight } \\
\text { severity }(\%)\end{array}$ & Summary & $\begin{array}{c}\text { Alternaria blight } \\
\text { incidence (\%) } \\
\text { (with inoculation) }\end{array}$ & Summary \\
\hline 1 & CIP 398203-2-1 & \begin{tabular}{|l|}
7.50 \\
$(2.83)$
\end{tabular} & MR & $\begin{array}{c}6.60 \\
(2.66)\end{array}$ & MR & $\begin{array}{c}22.90 \\
(28.60)\end{array}$ & MS \\
\hline 2 & CIP 398203-2-2 & $\begin{array}{c}5.90 \\
(2.44)\end{array}$ & MR & $\begin{array}{c}6.00 \\
(2.46)\end{array}$ & MR & $\begin{array}{c}21.00 \\
(27.19)\end{array}$ & MS \\
\hline 3 & CIP 398203-3-1 & $\begin{array}{c}5.10 \\
(2.37)\end{array}$ & MR & $\begin{array}{c}4.60 \\
(2.26)\end{array}$ & MR & $\begin{array}{c}22.20 \\
(28.06)\end{array}$ & MS \\
\hline 4 & CIP 302024-10-1 & $\begin{array}{c}6.50 \\
(2.65)\end{array}$ & MR & $\begin{array}{l}8.00 \\
(2.92)\end{array}$ & MR & $\begin{array}{l}11.90 \\
(20.19)\end{array}$ & MS \\
\hline 5 & CIP 302024-11-1 & $\begin{array}{c}4.40 \\
(2.21)\end{array}$ & MR & $\begin{array}{c}4.60 \\
(2.26)\end{array}$ & MR & $\begin{array}{c}12.30 \\
(20.48)\end{array}$ & MS \\
\hline 6 & CIP 302024-11-2 & $\begin{array}{c}5.80 \\
(2.51)\end{array}$ & MR & $\begin{array}{c}8.00 \\
(2.92)\end{array}$ & MR & $\begin{array}{c}10.00 \\
(18.29)\end{array}$ & MR \\
\hline 7 & CIP 302431-8-1 & $\begin{array}{c}2.60 \\
(1.76)\end{array}$ & MR & $\begin{array}{c}4.00 \\
(2.12)\end{array}$ & MR & $\begin{array}{c}12.70 \\
(20.79)\end{array}$ & MS \\
\hline 8 & CIP 302431-9-1 & $\begin{array}{c}4.20 \\
(2.17)\end{array}$ & MR & $\begin{array}{c}7.30 \\
(2.79)\end{array}$ & MR & $\begin{array}{c}11.70 \\
(19.85)\end{array}$ & MS \\
\hline 9 & CIP 304012-1-1 & $\begin{array}{l}3.70 \\
(2.05)\end{array}$ & MR & $\begin{array}{c}6.00 \\
(2.55)\end{array}$ & MR & $\begin{array}{c}12.60 \\
(20.75)\end{array}$ & MS \\
\hline 10 & CIP 304012-1-2 & $\begin{array}{l}4.10 \\
(2.14)\end{array}$ & MR & $\begin{array}{c}4.60 \\
(2.26)\end{array}$ & MR & $\begin{array}{c}10.60 \\
(18.92)\end{array}$ & MS \\
\hline 11 & CIP 303405-11-1 & $\begin{array}{l}3.20 \\
(1.92)\end{array}$ & MR & $\begin{array}{c}3.30 \\
(1.95)\end{array}$ & MR & $\begin{array}{c}22.00 \\
(27.91)\end{array}$ & MS \\
\hline 12 & CIP 303405-13-1 & $\begin{array}{c}3.20 \\
(1.92)\end{array}$ & MR & $\begin{array}{l}9.30 \\
(3.13)\end{array}$ & MR & $\begin{array}{c}21.90 \\
(27.88)\end{array}$ & MS \\
\hline 13 & CIP 303405-15-1 & $\begin{array}{c}3.30 \\
(1.95)\end{array}$ & MR & $\begin{array}{c}6.00 \\
(2.55)\end{array}$ & MR & $\begin{array}{c}22.60 \\
(28.32)\end{array}$ & MS \\
\hline 14 & CIP 303408-9-1 & $\begin{array}{c}4.50 \\
(2.24)\end{array}$ & MR & $\begin{array}{c}4.60 \\
(2.26)\end{array}$ & MR & $\begin{array}{c}14.80 \\
(22.56)\end{array}$ & MS \\
\hline 15 & CIP 303408-9-2 & $\begin{array}{l}4.10 \\
(2.14)\end{array}$ & MR & $\begin{array}{c}7.30 \\
(2.79)\end{array}$ & MR & $\begin{array}{c}15.60 \\
(23.10)\end{array}$ & MS \\
\hline 16 & CIP 303408-10-1 & $\begin{array}{c}4.20 \\
(2.17)\end{array}$ & MR & $\begin{array}{c}2.80 \\
(1.82)\end{array}$ & MR & $\begin{array}{c}15.90 \\
(23.52)\end{array}$ & MS \\
\hline 17 & CIP 304102-15-1 & $\begin{array}{c}4.10 \\
(2.14)\end{array}$ & MR & $\begin{array}{c}6.00 \\
(2.55)\end{array}$ & MR & $\begin{array}{l}20.40 \\
(26.77)\end{array}$ & MS \\
\hline 18 & CIP 304102-16-1 & $\begin{array}{c}5.20 \\
(2.39)\end{array}$ & MR & $\begin{array}{c}7.30 \\
(2.79)\end{array}$ & MR & $\begin{array}{c}21.80 \\
(27.79)\end{array}$ & MS \\
\hline 19 & CIP 304102-17-1 & $\begin{array}{c}3.90 \\
(2.10)\end{array}$ & MR & $\begin{array}{c}4.60 \\
(2.26)\end{array}$ & MR & $\begin{array}{c}19.50 \\
(26.90)\end{array}$ & MS \\
\hline 20 & CIP 304146-1-1 & $\begin{array}{r}7.80 \\
(2.88) \\
\end{array}$ & MR & $\begin{array}{r}8.00 \\
(2.92) \\
\end{array}$ & MR & $\begin{array}{c}23.20 \\
(28.72) \\
\end{array}$ & MS \\
\hline
\end{tabular}




\begin{tabular}{|c|c|c|c|c|c|c|c|}
\hline S. No. & Entries & $\begin{array}{c}\text { Alternaria } \\
\text { blight } \\
\text { incidence }(\%)\end{array}$ & Summary & $\begin{array}{c}\text { Alternaria } \\
\text { blight } \\
\text { severity }(\%)\end{array}$ & Summary & $\begin{array}{c}\text { Alternaria blight } \\
\text { incidence (\%) } \\
\text { (with inoculation) }\end{array}$ & Summary \\
\hline 21 & CIP 304146-2-1 & $\begin{array}{c}7.30 \\
(2.79)\end{array}$ & MR & $\begin{array}{l}12.60 \\
(3.62)\end{array}$ & MR & $\begin{array}{l}21.60 \\
(27.66)\end{array}$ & MS \\
\hline 22 & CIP 304146-3-1 & $\begin{array}{c}6.70 \\
(2.68)\end{array}$ & MR & $\begin{array}{c}8.60 \\
(3.02)\end{array}$ & MR & $\begin{array}{c}21.40 \\
(27.49)\end{array}$ & MS \\
\hline 23 & CIP 398201-19-1 & $\begin{array}{c}4.50 \\
(2.24)\end{array}$ & MR & $\begin{array}{c}5.30 \\
(2.41)\end{array}$ & MR & $\begin{array}{c}7.90 \\
(16.13)\end{array}$ & MR \\
\hline 24 & CIP 398201-19-2 & $\begin{array}{c}3.30 \\
(1.95)\end{array}$ & MR & $\begin{array}{c}4.00 \\
(2.12)\end{array}$ & MR & $\begin{array}{c}8.40 \\
(16.81)\end{array}$ & MR \\
\hline 25 & CIP 398201-20-1 & $\begin{array}{c}2.80 \\
(1.82)\end{array}$ & MR & $\begin{array}{c}3.30 \\
(1.95)\end{array}$ & MR & $\begin{array}{c}8.70 \\
(17.06)\end{array}$ & MR \\
\hline 26 & CIP 398201-22-1 & $\begin{array}{c}3.50 \\
(2.00)\end{array}$ & MR & $\begin{array}{c}6.60 \\
(2.66)\end{array}$ & MR & $\begin{array}{c}10.30 \\
(18.67)\end{array}$ & MS \\
\hline 27 & CIP 304014-1-1 & $\begin{array}{c}5.40 \\
(2.43)\end{array}$ & MR & $\begin{array}{l}9.30 \\
(3.13)\end{array}$ & MR & $\begin{array}{c}26.60 \\
(30.90)\end{array}$ & $\mathrm{S}$ \\
\hline 28 & CIP 304014-2-1 & $\begin{array}{c}5.20 \\
(2.39)\end{array}$ & MR & $\begin{array}{l}10.00 \\
(3.24)\end{array}$ & MR & $\begin{array}{c}25.20 \\
(30.09)\end{array}$ & $\mathrm{S}$ \\
\hline 29 & CIP 398181-1-1 & $\begin{array}{l}5.20 \\
(2.39)\end{array}$ & MR & $\begin{array}{c}7.30 \\
(2.79)\end{array}$ & MR & $\begin{array}{l}12.90 \\
(20.97)\end{array}$ & MS \\
\hline 30 & CIP 398181-2-1 & $\begin{array}{c}5.50 \\
(2.45)\end{array}$ & MR & $\begin{array}{l}8.00 \\
(2.92)\end{array}$ & MR & $\begin{array}{c}15.20 \\
(22.90)\end{array}$ & MS \\
\hline 31 & CIP 304124-14-1 & $\begin{array}{c}3.00 \\
(1.87)\end{array}$ & MR & $\begin{array}{c}9.30 \\
(3.13)\end{array}$ & MR & $\begin{array}{c}22.20 \\
(28.07)\end{array}$ & MS \\
\hline 32 & CIP 304124-15-1 & $\begin{array}{c}5.60 \\
(2.47)\end{array}$ & MR & $\begin{array}{c}8.00 \\
(2.92)\end{array}$ & MR & $\begin{array}{c}24.40 \\
(29.58)\end{array}$ & MS \\
\hline 33 & CIP 303139-11-1 & $\begin{array}{c}3.60 \\
(2.02)\end{array}$ & MR & $\begin{array}{c}5.30 \\
(2.41)\end{array}$ & MR & $\begin{array}{c}22.20 \\
(28.06)\end{array}$ & MS \\
\hline 34 & CIP 303139-12-1 & $\begin{array}{c}3.80 \\
(2.07)\end{array}$ & MR & $\begin{array}{c}6.00 \\
(2.55)\end{array}$ & MR & $\begin{array}{c}21.50 \\
(27.54)\end{array}$ & MS \\
\hline 35 & CIP 303139-13-1 & $\begin{array}{c}4.50 \\
(2.24)\end{array}$ & MR & $\begin{array}{c}4.60 \\
(2.26)\end{array}$ & MR & $\begin{array}{c}20.00 \\
(26.52)\end{array}$ & MS \\
\hline 36 & CIP 398068-19-1 & $\begin{array}{c}4.70 \\
(2.28)\end{array}$ & MR & $\begin{array}{c}6.60 \\
(2.66)\end{array}$ & MR & $\begin{array}{c}24.70 \\
(29.76)\end{array}$ & MS \\
\hline 37 & CIP 398068-21-1 & $\begin{array}{c}5.10 \\
(2.37)\end{array}$ & MR & $\begin{array}{r}7.30 \\
(2.79)\end{array}$ & MR & $\begin{array}{c}27.80 \\
(31.79)\end{array}$ & $\mathrm{S}$ \\
\hline 38 & CIP 398068-21-2 & $\begin{array}{c}4.60 \\
(2.26)\end{array}$ & MR & $\begin{array}{c}5.30 \\
(2.41)\end{array}$ & MR & $\begin{array}{c}24.50 \\
(29.62)\end{array}$ & MS \\
\hline 39 & CIP 398068-22-1 & $\begin{array}{c}5.20 \\
(2.39)\end{array}$ & MR & $\begin{array}{c}4.60 \\
(2.26)\end{array}$ & MR & $\begin{array}{c}22.80 \\
(28.49)\end{array}$ & MS \\
\hline 40 & KufriJawahar & $\begin{array}{c}2.60 \\
(1.76)\end{array}$ & MR & $\begin{array}{c}7.30 \\
(2.79)\end{array}$ & MR & $\begin{array}{c}33.90 \\
(26.71)\end{array}$ & $S$ \\
\hline 41 & KufriAshoka & $\begin{array}{c}3.50 \\
(2.00)\end{array}$ & MR & $\begin{array}{c}6.00 \\
(2.55)\end{array}$ & MR & $\begin{array}{c}21.60 \\
(27.40)\end{array}$ & MS \\
\hline
\end{tabular}




\begin{tabular}{|c|c|c|c|c|c|c|c|}
\hline S. No. & Entries & $\begin{array}{c}\text { Alternaria } \\
\text { blight } \\
\text { incidence }(\%)\end{array}$ & Summary & $\begin{array}{c}\text { Alternaria } \\
\text { blight } \\
\text { severity }(\%)\end{array}$ & Summary & $\begin{array}{c}\text { Alternaria blight } \\
\text { incidence (\%) } \\
\text { (with inoculation) }\end{array}$ & Summary \\
\hline 42 & KufriPukhraj & $\begin{array}{c}1.70 \\
(1.48)\end{array}$ & MR & $\begin{array}{c}6.00 \\
(2.55)\end{array}$ & MR & $\begin{array}{c}15.40 \\
(23.05)\end{array}$ & MS \\
\hline 43 & Kufri Surya & $\begin{array}{c}2.70 \\
(1.79)\end{array}$ & MR & $\begin{array}{c}4.00 \\
(2.12)\end{array}$ & MR & $\begin{array}{l}25.10 \\
(30.02)\end{array}$ & $S$ \\
\hline & $\begin{array}{l}\text { SEm } \pm \\
\text { C.D. (at 5\%) }\end{array}$ & $\begin{array}{c}0.232 \\
0.65 \\
\end{array}$ & & $\begin{array}{c}0.220 \\
0.79 \\
\end{array}$ & & $\begin{array}{c}1.876 \\
5.27 \\
\end{array}$ & \\
\hline
\end{tabular}

Average of three replications; (Data in parenthesis are transformed value)

$\mathrm{MR}=$ Moderately resistant $\mathrm{MS}=$ Moderately susceptible $\quad$ HR= Highly resistant

$\mathrm{SR}=$ Slightly resistant $\quad \mathrm{S}=$ Susceptible $\quad \mathrm{ScR}=$ Scarcely resistant

Table.4 Summary of disease reaction of $\mathrm{F}_{1} \mathrm{C}_{3}$ clonal progenies against Alternaria blight diseases

\begin{tabular}{|c|c|c|c|c|c|c|c|}
\hline $\begin{array}{l}\text { S. } \\
\text { No. }\end{array}$ & Entries & $\begin{array}{c}\text { Alternaria } \\
\text { blight } \\
\text { incidence }(\%)\end{array}$ & Summary & $\begin{array}{c}\text { Alternaria } \\
\text { blight } \\
\text { severity }(\%)\end{array}$ & Summary & \begin{tabular}{|c} 
Alternaria blight \\
incidence (\%) \\
(with \\
inoculation) \\
\end{tabular} & Summary \\
\hline 1 & CIP 398203-2-2-1 & $\begin{array}{c}2.30 \\
(1.67)\end{array}$ & MR & $\begin{array}{c}6.60 \\
(2.66)\end{array}$ & MR & $\begin{array}{c}21.90 \\
(27.81)\end{array}$ & MS \\
\hline 2 & CIP 398203-4-1-1 & $\begin{array}{c}2.70 \\
(1.79)\end{array}$ & MR & $\begin{array}{c}4.00 \\
(2.12)\end{array}$ & MR & $\begin{array}{c}24.60 \\
(29.69)\end{array}$ & MS \\
\hline 3 & CIP 398203-4-1-2 & $\begin{array}{c}2.90 \\
(1.84)\end{array}$ & MR & $\begin{array}{c}6.00 \\
(2.55)\end{array}$ & MR & $\begin{array}{c}23.60 \\
(29.04)\end{array}$ & MS \\
\hline 4 & CIP 398203-5-3-1 & $\begin{array}{c}3.50 \\
(2.00)\end{array}$ & MR & $\begin{array}{c}5.30 \\
(2.41)\end{array}$ & MR & $\begin{array}{c}23.50 \\
(28.95)\end{array}$ & MS \\
\hline 5 & CIP 398203-6-1-3 & $\begin{array}{c}3.10 \\
(1.90)\end{array}$ & MR & $\begin{array}{c}6.60 \\
(2.66)\end{array}$ & MR & $\begin{array}{c}22.00 \\
(27.86)\end{array}$ & MS \\
\hline 6 & CIP 398203-7-4-1 & $\begin{array}{c}2.40 \\
(1.70)\end{array}$ & MR & $\begin{array}{c}4.60 \\
(2.26)\end{array}$ & MR & $\begin{array}{c}22.40 \\
(28.21)\end{array}$ & MS \\
\hline 7 & CIP 398203-8-2-2 & $\begin{array}{c}2.50 \\
(1.73)\end{array}$ & MR & $\begin{array}{c}6.00 \\
(2.55)\end{array}$ & MR & $\begin{array}{c}24.30 \\
(29.47)\end{array}$ & MS \\
\hline 8 & CIP 302024-2-3-1 & $\begin{array}{c}2.40 \\
(1.70)\end{array}$ & MR & $\begin{array}{c}2.60 \\
(1.76)\end{array}$ & MR & $\begin{array}{l}12.10 \\
(20.29)\end{array}$ & MS \\
\hline 9 & CIP 302024-3-3-1 & $\begin{array}{c}3.20 \\
(1.92)\end{array}$ & MR & $\begin{array}{c}4.60 \\
(2.26)\end{array}$ & MR & $\begin{array}{c}13.40 \\
(21.44)\end{array}$ & MS \\
\hline 10 & CIP 302024-3-4-2 & $\begin{array}{l}3.70 \\
(2.05)\end{array}$ & MR & $\begin{array}{c}8.60 \\
(3.02)\end{array}$ & MR & $\begin{array}{c}11.80 \\
(20.12)\end{array}$ & MS \\
\hline 11 & CIP 302024-5-1-1 & $\begin{array}{c}5.50 \\
(2.45)\end{array}$ & MR & $\begin{array}{c}7.30 \\
(2.79)\end{array}$ & MR & $\begin{array}{c}13.10 \\
(21.07)\end{array}$ & MS \\
\hline 12 & CIP 302024-6-2-1 & $\begin{array}{c}5.40 \\
(2.43)\end{array}$ & MR & $\begin{array}{c}8.00 \\
(2.92)\end{array}$ & MR & $\begin{array}{c}11.50 \\
(19.77)\end{array}$ & MS \\
\hline 13 & CIP 302431-2-1-1 & $\begin{array}{c}1.80 \\
(1.52)\end{array}$ & MR & $\begin{array}{c}5.30 \\
(2.41)\end{array}$ & MR & $\begin{array}{c}13.00 \\
(20.96)\end{array}$ & MS \\
\hline 14 & CIP 302431-2-3-2 & 2.50 & MR & 6.60 & MR & 13.40 & MS \\
\hline
\end{tabular}




\begin{tabular}{|c|c|c|c|c|c|c|c|}
\hline $\begin{array}{c}\text { S. } \\
\text { No. }\end{array}$ & Entries & $\begin{array}{c}\text { Alternaria } \\
\text { blight } \\
\text { incidence( } \%)\end{array}$ & Summary & $\begin{array}{c}\text { Alternaria } \\
\text { blight } \\
\text { severity }(\%)\end{array}$ & Summary & \begin{tabular}{|c|} 
Alternaria blight \\
incidence $(\%)$ \\
(with \\
inoculation) \\
\end{tabular} & Summary \\
\hline 15 & CIP 302431-3-2-1 & $\begin{array}{c}(1.73) \\
3.60 \\
(2.02)\end{array}$ & MR & $\begin{array}{l}(2.66) \\
5.30 \\
(2.41)\end{array}$ & MR & $\begin{array}{l}(21.19) \\
13.60 \\
(21.52)\end{array}$ & MS \\
\hline 16 & CIP 302431-4-1-1 & $\begin{array}{c}2.30 \\
(1.67)\end{array}$ & MR & $\begin{array}{c}6.60 \\
(2.66)\end{array}$ & MR & $\begin{array}{l}15.20 \\
(22.86)\end{array}$ & MS \\
\hline 17 & CIP 302431-5-3-2 & $\begin{array}{c}4.40 \\
(2.21)\end{array}$ & MR & $\begin{array}{c}8.00 \\
(2.92)\end{array}$ & MR & $\begin{array}{c}13.80 \\
(12.60)\end{array}$ & MS \\
\hline 18 & CIP 302431-6-4-1 & $\begin{array}{c}4.60 \\
(2.26)\end{array}$ & MR & $\begin{array}{c}7.30 \\
(2.79)\end{array}$ & MR & $\begin{array}{c}15.60 \\
(23.18)\end{array}$ & MS \\
\hline 19 & CIP 304012-1-4-1 & $\begin{array}{c}5.10 \\
(2.37)\end{array}$ & MR & $\begin{array}{l}14.60 \\
(3.89)\end{array}$ & MS & $\begin{array}{c}12.70 \\
(20.79)\end{array}$ & MS \\
\hline 20 & CIP 304012-6-1-1 & $\begin{array}{c}3.40 \\
(1.97)\end{array}$ & MR & $\begin{array}{c}6.60 \\
(2.66)\end{array}$ & MR & $\begin{array}{c}13.00 \\
(20.99)\end{array}$ & MS \\
\hline 21 & CIP 304012-6-2-3 & $\begin{array}{c}1.60 \\
(1.45)\end{array}$ & MR & $\begin{array}{c}4.00 \\
(2.12)\end{array}$ & MR & $\begin{array}{c}10.80 \\
(19.16)\end{array}$ & MS \\
\hline 22 & CIP 304012-7-3-1 & $\begin{array}{c}3.60 \\
(2.02)\end{array}$ & MR & $\begin{array}{c}7.30 \\
(2.79)\end{array}$ & MR & $\begin{array}{c}15.40 \\
(23.09)\end{array}$ & MS \\
\hline 23 & CIP 304012-12-4-1 & $\begin{array}{c}4.80 \\
(2.30)\end{array}$ & MR & $\begin{array}{l}13.30 \\
(3.71)\end{array}$ & MS & $\begin{array}{c}13.00 \\
(20.91)\end{array}$ & MS \\
\hline 24 & CIP 304012-9-2-1 & $\begin{array}{c}4.30 \\
(2.19)\end{array}$ & MR & $\begin{array}{l}10.00 \\
(3.24)\end{array}$ & MR & $\begin{array}{c}14.10 \\
(21.89)\end{array}$ & MS \\
\hline 25 & CIP 303405-4-2-2 & $\begin{array}{c}3.10 \\
(1.90)\end{array}$ & MR & $\begin{array}{c}7.30 \\
(2.79)\end{array}$ & MR & $\begin{array}{c}23.50 \\
(28.99)\end{array}$ & MS \\
\hline 26 & CIP 303405-3-1-1 & $\begin{array}{c}1.90 \\
(1.55)\end{array}$ & MR & $\begin{array}{c}3.30 \\
(1.95)\end{array}$ & MR & $\begin{array}{c}21.20 \\
(27.31)\end{array}$ & MS \\
\hline 27 & CIP 303405-3-1-2 & $\begin{array}{c}3.40 \\
(1.97)\end{array}$ & MR & $\begin{array}{l}5.30 \\
(2.41)\end{array}$ & MR & $\begin{array}{c}22.20 \\
(28.06)\end{array}$ & MS \\
\hline 28 & CIP 303405-8-3-1 & $\begin{array}{c}5.80 \\
(2.51)\end{array}$ & MR & $\begin{array}{l}12.00 \\
(3.54)\end{array}$ & MS & $\begin{array}{c}23.00 \\
(29.07)\end{array}$ & MS \\
\hline 29 & CIP 303405-6-2-1 & $\begin{array}{c}1.60 \\
(1.45)\end{array}$ & MR & $\begin{array}{c}7.30 \\
(2.79)\end{array}$ & MR & $\begin{array}{l}23.10 \\
(28.64)\end{array}$ & MS \\
\hline 30 & CIP 303405-7-4-1 & $\begin{array}{c}1.90 \\
(1.55)\end{array}$ & MR & $\begin{array}{c}6.00 \\
(2.55)\end{array}$ & MR & $\begin{array}{c}25.60 \\
(30.38)\end{array}$ & $S$ \\
\hline 31 & CIP 303405-9-1-1 & $\begin{array}{c}3.10 \\
(1.90)\end{array}$ & MR & $\begin{array}{c}5.30 \\
(2.41)\end{array}$ & MR & $\begin{array}{c}23.30 \\
(28.72)\end{array}$ & MS \\
\hline 32 & CIP 303405-5-3-1 & $\begin{array}{c}1.00 \\
(1.22)\end{array}$ & MR & $\begin{array}{c}3.30 \\
(1.95)\end{array}$ & MR & $\begin{array}{c}25.40 \\
(30.22)\end{array}$ & S \\
\hline 33 & CIP 303408-6-3-1 & $\begin{array}{c}2.90 \\
(1.84)\end{array}$ & MR & $\begin{array}{c}8.00 \\
(2.92)\end{array}$ & MR & $\begin{array}{c}13.60 \\
(21.53)\end{array}$ & MS \\
\hline 34 & CIP 303408-8-2-2 & $\begin{array}{c}2.50 \\
(1.73)\end{array}$ & MR & $\begin{array}{c}5.30 \\
(2.41)\end{array}$ & MR & $\begin{array}{c}16.90 \\
(24.22)\end{array}$ & MS \\
\hline
\end{tabular}




\begin{tabular}{|c|c|c|c|c|c|c|c|}
\hline $\begin{array}{c}\text { S. } \\
\text { No. }\end{array}$ & Entries & $\begin{array}{c}\text { Alternaria } \\
\text { blight } \\
\text { incidence( } \%)\end{array}$ & Summary & $\begin{array}{c}\text { Alternaria } \\
\text { blight } \\
\text { severity }(\%)\end{array}$ & Summary & \begin{tabular}{|c|} 
Alternaria blight \\
incidence $(\%)$ \\
(with \\
inoculation) \\
\end{tabular} & Summary \\
\hline 35 & CIP 303408-8-3-1 & $\begin{array}{c}4.70 \\
(2.28)\end{array}$ & MR & $\begin{array}{l}10.00 \\
(3.24)\end{array}$ & MR & $\begin{array}{c}15.30 \\
(22.96)\end{array}$ & MS \\
\hline 36 & CIP 303408-4-5-1 & $\begin{array}{c}4.00 \\
(2.12)\end{array}$ & MR & $\begin{array}{l}10.60 \\
(3.33)\end{array}$ & MS & $\begin{array}{c}15.40 \\
(23.06)\end{array}$ & MS \\
\hline 37 & CIP 303408-2-1-1 & $\begin{array}{c}4.10 \\
(2.14)\end{array}$ & MR & $\begin{array}{l}14.00 \\
(3.81)\end{array}$ & MS & $\begin{array}{c}16.40 \\
(23.83)\end{array}$ & MS \\
\hline 38 & CIP 303408-7-3-1 & $\begin{array}{c}2.90 \\
(1.84)\end{array}$ & MR & $\begin{array}{l}12.00 \\
(3.54)\end{array}$ & MS & $\begin{array}{c}19.00 \\
(25.78)\end{array}$ & MS \\
\hline 39 & CIP 303408-3-2-1 & $\begin{array}{c}2.40 \\
(1.70)\end{array}$ & MR & $\begin{array}{c}8.00 \\
(2.92)\end{array}$ & MR & $\begin{array}{c}16.40 \\
(23.80)\end{array}$ & MS \\
\hline 40 & CIP 304102-11-1-2 & $\begin{array}{c}4.90 \\
(2.32)\end{array}$ & MR & $\begin{array}{l}12.60 \\
(3.62)\end{array}$ & MS & $\begin{array}{c}18.20 \\
(25.10)\end{array}$ & MS \\
\hline 41 & CIP 304102-9-3-1 & $\begin{array}{c}6.60 \\
(2.66)\end{array}$ & MR & $\begin{array}{l}16.60 \\
(4.14)\end{array}$ & MS & $\begin{array}{c}12.70 \\
(20.81)\end{array}$ & MS \\
\hline 42 & CIP 304102-4-2-1 & $\begin{array}{c}6.90 \\
(2.72)\end{array}$ & MR & $\begin{array}{c}8.00 \\
(2.92)\end{array}$ & MR & $\begin{array}{c}13.90 \\
(21.81)\end{array}$ & MS \\
\hline 43 & CIP 304102-4-5-1 & $\begin{array}{c}5.10 \\
(2.37)\end{array}$ & MR & $\begin{array}{l}11.30 \\
(3.44)\end{array}$ & MS & $\begin{array}{c}19.80 \\
(26.36)\end{array}$ & MS \\
\hline 44 & CIP 304102-7-1-1 & $\begin{array}{c}7.10 \\
(2.76)\end{array}$ & MR & $\begin{array}{l}10.00 \\
(3.24)\end{array}$ & MR & $\begin{array}{c}18.30 \\
(25.29)\end{array}$ & MS \\
\hline 45 & CIP 304146-12-4-1 & $\begin{array}{c}7.30 \\
(2.79)\end{array}$ & MR & $\begin{array}{c}6.60 \\
(2.66)\end{array}$ & MR & $\begin{array}{c}25.10 \\
(29.95)\end{array}$ & $S$ \\
\hline 46 & CIP 304146-11-2-2 & $\begin{array}{c}7.20 \\
(2.77)\end{array}$ & MR & $\begin{array}{c}8.60 \\
(3.02)\end{array}$ & MR & $\begin{array}{c}24.80 \\
(29.83)\end{array}$ & MS \\
\hline 47 & CIP 304146-11-3-1 & $\begin{array}{c}5.50 \\
(2.45)\end{array}$ & MR & $\begin{array}{l}17.30 \\
(4.22)\end{array}$ & MS & $\begin{array}{c}22.20 \\
(28.04)\end{array}$ & MS \\
\hline 48 & CIP 304146-1-4-1 & $\begin{array}{c}6.50 \\
(2.65)\end{array}$ & MR & $\begin{array}{l}13.30 \\
(3.71)\end{array}$ & MS & $\begin{array}{c}25.40 \\
(30.23)\end{array}$ & $S$ \\
\hline 49 & CIP 304146-2-2-1 & $\begin{array}{c}7.70 \\
(2.86)\end{array}$ & MR & $\begin{array}{c}9.30 \\
(3.13)\end{array}$ & MR & $\begin{array}{c}24.40 \\
(29.59)\end{array}$ & MS \\
\hline 50 & CIP 398201-3-3-1 & $\begin{array}{c}4.50 \\
(2.24)\end{array}$ & MR & $\begin{array}{c}6.60 \\
(2.66)\end{array}$ & MR & $\begin{array}{c}11.50 \\
(19.78)\end{array}$ & MS \\
\hline 51 & CIP 398201-3-3-2 & $\begin{array}{c}5.60 \\
(2.47)\end{array}$ & MR & $\begin{array}{c}9.30 \\
(3.13)\end{array}$ & MR & $\begin{array}{c}12.80 \\
(20.88)\end{array}$ & MS \\
\hline 52 & CIP 398201-7-2-1 & $\begin{array}{c}4.00 \\
(2.12)\end{array}$ & MR & $\begin{array}{c}3.30 \\
(1.95)\end{array}$ & MR & $\begin{array}{c}9.60 \\
(17.96)\end{array}$ & MR \\
\hline 53 & CIP 398201-15-4-1 & $\begin{array}{c}5.00 \\
(2.35)\end{array}$ & MR & $\begin{array}{c}8.00 \\
(2.92)\end{array}$ & MR & $\begin{array}{c}8.30 \\
(16.71)\end{array}$ & MR \\
\hline 54 & CIP 398201-5-3-1 & $\begin{array}{c}3.60 \\
(2.02)\end{array}$ & MR & $\begin{array}{c}7.30 \\
(2.79)\end{array}$ & MR & $\begin{array}{c}9.50 \\
(17.89)\end{array}$ & MR \\
\hline 55 & CIP 398201-11-6-1 & 5.30 & MR & 8.00 & MR & 9.50 & MR \\
\hline
\end{tabular}




\begin{tabular}{|c|c|c|c|c|c|c|c|}
\hline $\begin{array}{c}\text { S. } \\
\text { No. }\end{array}$ & Entries & $\begin{array}{c}\text { Alternaria } \\
\text { blight } \\
\text { incidence }(\%)\end{array}$ & Summary & $\begin{array}{c}\text { Alternaria } \\
\text { blight } \\
\text { severity }(\%)\end{array}$ & Summary & \begin{tabular}{|c|} 
Alternaria blight \\
incidence $(\%)$ \\
(with \\
inoculation) \\
\end{tabular} & Summary \\
\hline 56 & CIP 398201-2-2-1 & $\begin{array}{l}(2.41) \\
5.60 \\
(2.47)\end{array}$ & MR & $\begin{array}{l}(2.92) \\
7.30 \\
(2.79)\end{array}$ & MR & $\begin{array}{c}(17.78) \\
8.40 \\
(16.77)\end{array}$ & MR \\
\hline 57 & CIP 304014-8-4-2 & $\begin{array}{c}5.20 \\
(2.39)\end{array}$ & MR & $\begin{array}{c}6.00 \\
(2.55)\end{array}$ & MR & $\begin{array}{c}28.30 \\
(32.09)\end{array}$ & S \\
\hline 58 & CIP 304014-2-6-1 & $\begin{array}{c}4.60 \\
(2.26)\end{array}$ & MR & $\begin{array}{c}4.00 \\
(2.12)\end{array}$ & MR & $\begin{array}{c}22.70 \\
(28.41)\end{array}$ & MS \\
\hline 59 & CIP 304014-1-3-1 & $\begin{array}{c}5.00 \\
(2.35)\end{array}$ & MR & $\begin{array}{c}6.60 \\
(2.66)\end{array}$ & MR & $\begin{array}{c}23.00 \\
(28.63)\end{array}$ & MS \\
\hline 60 & CIP 304014-12-2-1 & $\begin{array}{l}4.20 \\
(2.17)\end{array}$ & MR & $\begin{array}{c}5.30 \\
(2.41)\end{array}$ & MR & $\begin{array}{c}24.00 \\
(29.32)\end{array}$ & MS \\
\hline 61 & CIP 304014-5-4-1 & $\begin{array}{c}4.20 \\
(2.17)\end{array}$ & MR & $\begin{array}{c}6.00 \\
(2.55)\end{array}$ & MR & $\begin{array}{l}23.00 \\
(28.64)\end{array}$ & MS \\
\hline 62 & CIP 304014-9-2-1 & $\begin{array}{l}5.60 \\
(2.47)\end{array}$ & MR & $\begin{array}{c}8.60 \\
(3.02)\end{array}$ & MR & $\begin{array}{c}28.00 \\
(31.91)\end{array}$ & $S$ \\
\hline 63 & CIP 398181-9-3-1 & $\begin{array}{c}4.90 \\
(2.32)\end{array}$ & MR & $\begin{array}{c}8.60 \\
(3.02)\end{array}$ & MR & $\begin{array}{c}15.50 \\
(23.19)\end{array}$ & MS \\
\hline 64 & CIP 398181-17-2-1 & $\begin{array}{c}5.50 \\
(2.45)\end{array}$ & MR & $\begin{array}{c}8.60 \\
(3.02)\end{array}$ & MR & $\begin{array}{l}16.70 \\
(24.05)\end{array}$ & MS \\
\hline 65 & CIP 398181-14-5-1 & $\begin{array}{c}5.60 \\
(2.47)\end{array}$ & MR & $\begin{array}{c}7.30 \\
(2.79)\end{array}$ & MR & $\begin{array}{c}14.60 \\
(22.35)\end{array}$ & MS \\
\hline 66 & CIP 398181-7-4-1 & $\begin{array}{c}4.60 \\
(2.26)\end{array}$ & MR & $\begin{array}{c}8.60 \\
(3.02)\end{array}$ & MR & $\begin{array}{c}17.70 \\
(24.88)\end{array}$ & MS \\
\hline 67 & CIP 398181-10-1-1 & $\begin{array}{c}4.70 \\
(2.28)\end{array}$ & MR & $\begin{array}{l}8.00 \\
(2.92)\end{array}$ & MR & $\begin{array}{c}14.80 \\
(22.61)\end{array}$ & MS \\
\hline 68 & CIP 398181-16-5-1 & $\begin{array}{c}3.00 \\
(1.87)\end{array}$ & MR & $\begin{array}{c}7.30 \\
(2.79)\end{array}$ & MR & $\begin{array}{c}16.00 \\
(23.47)\end{array}$ & MS \\
\hline 69 & CIP 304124-2-5-1 & $\begin{array}{c}6.80 \\
(2.70)\end{array}$ & MR & $\begin{array}{c}9.30 \\
(3.13)\end{array}$ & MR & $\begin{array}{l}21.90 \\
(27.84)\end{array}$ & MS \\
\hline 70 & CIP 304124-7-2-1 & $\begin{array}{c}7.50 \\
(2.83)\end{array}$ & MR & $\begin{array}{l}12.00 \\
(3.54)\end{array}$ & MS & $\begin{array}{c}25.50 \\
(30.33)\end{array}$ & $S$ \\
\hline 71 & CIP 304124-8-3-1 & $\begin{array}{c}5.60 \\
(2.47)\end{array}$ & MR & $\begin{array}{l}13.30 \\
(3.71)\end{array}$ & MS & $\begin{array}{c}23.50 \\
(28.89)\end{array}$ & MS \\
\hline 72 & CIP 304124-1-4-1 & $\begin{array}{c}6.20 \\
(2.59)\end{array}$ & MR & $\begin{array}{l}12.00 \\
(3.54)\end{array}$ & MS & $\begin{array}{c}22.10 \\
(27.97)\end{array}$ & MS \\
\hline 73 & CIP 304124-4-3-1 & $\begin{array}{c}5.40 \\
(2.43)\end{array}$ & MR & $\begin{array}{l}12.00 \\
(3.54)\end{array}$ & MS & $\begin{array}{c}21.90 \\
(27.83)\end{array}$ & MS \\
\hline 74 & CIP 303139-4-4-1 & $\begin{array}{c}3.80 \\
(2.07)\end{array}$ & MR & $\begin{array}{c}5.30 \\
(2.41)\end{array}$ & MR & $\begin{array}{c}18.40 \\
(25.38)\end{array}$ & MS \\
\hline 75 & CIP 303139-9-2-1 & $\begin{array}{l}4.80 \\
(2.30)\end{array}$ & MR & $\begin{array}{l}4.60 \\
(2.26)\end{array}$ & MR & $\begin{array}{c}21.90 \\
(27.87)\end{array}$ & MS \\
\hline
\end{tabular}




\begin{tabular}{|c|c|c|c|c|c|c|c|}
\hline $\begin{array}{c}\text { S. } \\
\text { No. }\end{array}$ & Entries & $\begin{array}{c}\text { Alternaria } \\
\text { blight } \\
\text { incidence }(\%)\end{array}$ & Summary & $\begin{array}{c}\text { Alternaria } \\
\text { blight } \\
\text { severity }(\%)\end{array}$ & Summary & \begin{tabular}{|c|}
$\begin{array}{c}\text { Alternaria blight } \\
\text { incidence }(\%) \\
\text { (with } \\
\text { inoculation) }\end{array}$ \\
\end{tabular} & Summary \\
\hline 76 & CIP 303139-9-4-1 & $\begin{array}{c}5.20 \\
(2.39)\end{array}$ & MR & $\begin{array}{c}5.30 \\
(2.41)\end{array}$ & MR & $\begin{array}{c}20.30 \\
(26.71)\end{array}$ & MS \\
\hline 77 & CIP 303139-2-2-1 & $\begin{array}{c}5.10 \\
(2.37)\end{array}$ & MR & $\begin{array}{c}6.00 \\
(2.55)\end{array}$ & MR & $\begin{array}{c}21.80 \\
(27.78)\end{array}$ & MS \\
\hline 78 & CIP 303139-8-3-1 & $\begin{array}{c}5.60 \\
(2.47)\end{array}$ & MR & $\begin{array}{c}5.30 \\
(2.41)\end{array}$ & MR & $\begin{array}{c}21.20 \\
(27.24)\end{array}$ & MS \\
\hline 79 & CIP 303139-10-3-1 & $\begin{array}{c}4.80 \\
(2.30)\end{array}$ & MR & $\begin{array}{c}4.00 \\
(2.12)\end{array}$ & MR & $\begin{array}{c}25.40 \\
(30.21)\end{array}$ & S \\
\hline 80 & CIP 398068-15-3-1 & $\begin{array}{c}4.20 \\
(2.17)\end{array}$ & MR & $\begin{array}{c}4.60 \\
(2.26)\end{array}$ & MR & $\begin{array}{c}26.10 \\
(30.72)\end{array}$ & S \\
\hline 81 & CIP 398068-15-3-2 & $\begin{array}{c}5.50 \\
(2.45)\end{array}$ & MR & $\begin{array}{c}6.60 \\
(2.66)\end{array}$ & MR & $\begin{array}{c}23.20 \\
(28.78)\end{array}$ & MS \\
\hline 82 & CIP 398068-3-2-1 & $\begin{array}{c}5.00 \\
(2.35)\end{array}$ & MR & $\begin{array}{c}0.30 \\
(0.89)\end{array}$ & MR & $\begin{array}{c}20.40 \\
(26.77)\end{array}$ & MS \\
\hline 83 & CIP 398068-9-4-1 & $\begin{array}{c}5.50 \\
(2.45)\end{array}$ & MR & $\begin{array}{l}12.60 \\
(3.62)\end{array}$ & MS & $\begin{array}{c}21.70 \\
(27.67)\end{array}$ & MS \\
\hline 84 & CIP 398068-1-5-1 & $\begin{array}{c}6.60 \\
(2.66)\end{array}$ & MR & $\begin{array}{l}13.30 \\
(3.71)\end{array}$ & MS & $\begin{array}{c}21.80 \\
(27.77)\end{array}$ & MS \\
\hline 85 & CIP 398068-4-2-1 & $\begin{array}{c}5.50 \\
(2.45)\end{array}$ & MR & $\begin{array}{c}7.30 \\
(2.79)\end{array}$ & MR & $\begin{array}{c}23.50 \\
(28.99)\end{array}$ & MS \\
\hline 86 & CIP 398068-7-3-1 & $\begin{array}{c}5.60 \\
(2.47)\end{array}$ & MR & $\begin{array}{c}4.60 \\
(2.26)\end{array}$ & MR & $\begin{array}{c}22.20 \\
(28.05)\end{array}$ & MS \\
\hline 87 & KufriJawahar & $\begin{array}{c}2.40 \\
(1.70)\end{array}$ & MR & $\begin{array}{c}6.00 \\
(2.55)\end{array}$ & MR & $\begin{array}{c}23.70 \\
(29.07)\end{array}$ & MS \\
\hline 88 & KufriAshoka & $\begin{array}{c}2.40 \\
(1.70)\end{array}$ & MR & $\begin{array}{c}7.30 \\
(2.79)\end{array}$ & MR & $\begin{array}{c}30.30 \\
(33.38)\end{array}$ & S \\
\hline 89 & KufriPukhraj & $\begin{array}{c}2.00 \\
(1.58)\end{array}$ & MR & $\begin{array}{c}4.60 \\
(2.26)\end{array}$ & MR & $\begin{array}{c}16.60 \\
(24.02)\end{array}$ & MS \\
\hline \multirow[t]{2}{*}{90} & Kufri Surya & 2.50 & \multirow[t]{2}{*}{ MR } & 7.30 & \multirow[t]{2}{*}{ MR } & & MS \\
\hline & $\begin{array}{l}\text { SEm } \pm \\
\text { C.D. (at } 5 \% \text { ) }\end{array}$ & $\begin{array}{c}(1.73) \\
0.257 \\
0.71 \\
\end{array}$ & & $\begin{array}{c}(2.79) \\
0.326 \\
0.91 \\
\end{array}$ & & $\begin{array}{c}(29.26) \\
1.422 \\
3.94 \\
\end{array}$ & MS \\
\hline
\end{tabular}

Average of three replications; (Data in parenthesis are transformed value)

$\mathrm{MR}=$ Moderately resistant $\mathrm{MS}=$ Moderately susceptible $\mathrm{HR}=$ Highly resistant

$\mathrm{SR}=$ Slightly resistant $\quad \mathrm{S}=$ Susceptible $\quad \mathrm{ScR}=$ Scarcely resistant

a) Evaluation of bulks, $\mathrm{F}_{1} \mathrm{C}_{2}$ and $\mathrm{F}_{1} \mathrm{C}_{3}$ clonal progenies against Alternaria blight disease with artificial inoculation

Per cent disease incidence under artificial inoculation condition were recorded and presented in Table 1 for potato bulks, the minimum blight incidence was observed in CIP 398201 (7.60\%) followed by CIP 304012 (10.80\%), CIP 302024 (11.60\%), CIP 398181 (12.90\%) and CIP 302431 (13.20\%). The maximum disease incidence was recorded in 
KufriJawahar $(36.70 \%)$ followed by CIP 304014 (26.60\%), Kufri Surya (24.70\%), KufriAshoka (23.70\%), CIP 398203 (22.90\%), CIP 303405 (22.10\%), CIP 303139 (21.90\%) and CIP 398068 (21.70\%).

Similarly, the entries of $\mathrm{F}_{1} \mathrm{C}_{2}$ clonal progenies (Table 2) recorded minimum per cent incidence in CIP 398201-19-1 (7.90\%) followed by CIP 398201-19-2 (8.40\%), CIP 398201-20-1 (8.70\%) and CIP 302024-11-2 $(10.0 \%)$ whereas the maximum incidence was observed in entry KufriJawahar (33.90\%) followed by CIP 398068-21-1 (27.80\%), CIP 304014-1-1 (26.60\%), CIP 304014-2-1 (25.20\%), Kufri Surya (25.10\%), CIP 398068-19-1 (24.70\%), CIP 398068-21-2 (24.50\%) and CIP 304124-15-1 (24.40\%).

Whereas, the incidence of Alternaria blight in $\mathrm{F}_{1} \mathrm{C}_{3}$ clonal progenies (Table 3) of potato ranged from 8.30 per cent to 30.30 per cent and minimum incidence was observed in CIP 398201-15-4-1 (8.30\%) followed by CIP 398201-2-2-1 (8.40\%), CIP 398201-5-3-1 (9.50\%), CIP 898201-11-6-1 (9.50\%) and CIP 398201-7-2-1 (9.60\%). The maximum disease incidence of 30.30 per cent was recorded in KufriAshoka, followed by CIP 304014-8-4-2 (28.30\%) and CIP 304014-9-2-1 (28.0\%). The rest of the entries were recorded to be statistically at par.

Various levels of reactions were observed among the artificial inoculated genotypes. The genotype CIP 398201 was found moderately resistant and two genotypes viz., CIP 304014 and KufriJawahar were susceptible against blight resistance and rest of the progenies showed moderate susceptible reaction. In $\mathrm{F}_{1} \mathrm{C}_{2}$ clonal progenies the genotype CIP 302024-11-2, CIP 398201-191, CIP 398201-19-2 and CIP 398201-20-1 showed moderately resistant reaction and rest of the progenies were found moderately susceptible (34 progenies) and susceptible (5) against blight. Similarly, in $\mathrm{F}_{1} \mathrm{C}_{3}$ clonal progenies, genotypes CIP 398201-7-2-1, CIP 398201-15-4-1, CIP 398201-5-3-1, CIP 398201-11-6-1 and CIP 398201-2-2-1 were found moderately resistant .While, 12 progenies showed susceptible reaction and rest of the 73 progenies showed moderately susceptible reaction against blight resistance (Tiwari et al., 2004; Kang et al., 2007; Kumar et al., 2009).

It can be concluded, on the basis of screening, all the genotypes of clonal bulks, $\mathrm{F}_{1} \mathrm{C}_{2}$ and $\mathrm{F}_{1} \mathrm{C}_{3}$ progenies showed moderate resistance reaction against blight incidence and most of the genotypes of clonal bulks, $\mathrm{F}_{1} \mathrm{C}_{2}$ and $\mathrm{F}_{1} \mathrm{C}_{3}$ clonal progenies showed moderate resistance against blight severity. Among artificial inoculated genotypes of clonal bulks and progenies the genotype CIP 398201 of clonal bulk, four genotypes (CIP 302024-11-2, CIP 398201-19-1, CIP 398201-19-2 and CIP 398201-20-1) of $\mathrm{F}_{1} \mathrm{C}_{2}$ progenies and five genotypes (CIP 398201-7-2-1, CIP 39820115-4-1, CIP 398201-5-3-1, CIP 398201-11-61 and CIP 398201-2-2-1) of $\mathrm{F}_{1} \mathrm{C}_{3}$ progenies were found moderately resistant against blight incidence. Most of the remaining genotypes showed moderately susceptible reaction.

Screening against Alternaria blight revealed that most of the genotypes showed moderate resistance against blight incidence and blight severity. But among artificially inoculated genotypes most of them showed moderately susceptible reaction against blight incidence.

\section{References}

Chirst, Haynes. 2001. Inheritance of resistance to early blight disease in a diploid potato population. Plant Breed., 120(2): 169-172.

Edin, E., Andersson, B. 2014. The early blight situation in Sweden - species abundance and strobilurin sensitivity. In: Schepers 
$\mathrm{H}$, eds. Proceedings of the 14th EuroBlight Workshop. Limassol, Cyprus: Wageningen UR, pp. 83-84

Kang, G.S., Kumar, R., Gopal, J., Pandey, S.K. and Paul Khurana, S.M. 2007. KufriPushkar - A main crop potato variety with good keeping quality for Indian plains. Potato J., 34(3-4): 147152.

Kapsa, J.S. and Osowski, J. 2012. Hostpathogen interaction between Alternaria species and $\mathrm{S}$. tuberosum under different conditions. Special Report No. 15: pp. 107.

Kumar, R., Kang G.S., Pandey, S.K. and Gopal, J. 2009. KufriKhyati: A new early maturing potato variety for Indian Plains. Potato J., 36(1-2): 14-19.

Leiminger, J.H. and Hausladen, H. 2012. Early Blight Control in Potato Using Disease-Orientated Threshold Values. Plant Dis., 96(1): pp. 124-130.

Malik, S.A. and M.A. Khan. 1967. Investigations on the control of early blight of potato caused by Alternaria
solani(Ellis and Mart) Jones and Grout. W. Pak. J. Agric. Res., 5: 92-97.

Pandey, S.K., Singh, S.V., Kumar, D., Manivel, P., Marwaha, R.S., Kumar, P. and Singh, B.P. 2006. Kufri Chipsona3: A high yielding potato variety for chipping with defect free tubers. Potato J., 33(1-2): 26-34.

Runno-Paurson, E., Loit, K., Hansen, M., Tein, B., Williams, I.H. and Maend, M. 2015. Early blight destroys potato foliage in the northern Baltic region. Acta Agriculturae Scandinavica Section B, Soil and Plant Sci., 65(5): pp. 422432.

Stuart, W. 1923. The potato. J.B. Limpincott. Co. Philadelphia, p. 249-251.

Tiwari, R.K.S., Rajput, M.L. and Singh, A. 2004. Effect of sowing dates and spray schedule of mancozeb on early blight Alternaria solani [(Ell. and Mart.) Jones and Grout] of potato. Indian J. Plant Protection, 32(2): 61-64.

Whetzel, H.H. 1923. The Alternaria blight of potatoes in Bermuda. Phytopathol., 13: $100-103$.

\section{How to cite this article:}

Nirmodh Prabha and Nanda, H.C. 2017. Screening for Alternaria Blight in Clonal Progenies of Potato (Solanum tuberosum L.). Int.J.Curr.Microbiol.App.Sci. 6(3): 1379-1391. doi: https://doi.org/10.20546/ijcmas.2017.603.160 